\title{
O Perdão sob a Perspectiva do Ofensor: uma Revisão da Sistemática
}

\author{
Thalita Fernandes Alencar \\ Universidade Federal da Paraíba, PB, Brasil.
}

\author{
Eloá Losano Abreu \\ Universidade Federal da Paraíba, PB, Brasil.
}

Resumo: Neste estudo foram realizadas revisões sistemáticas sobre as atitudes para receber o perdão e para perdoar a si mesmo. Considerando a escassez de estudos e relevância dos temas, 0 objetivo foi conhecer a forma como essas atitudes foram investigadas nas publicações recentes. O levantamento dos artigos foi realizado no portal Periódicos Capes, sendo selecionados estudos publicados entre 2006 e 2016. Na revisão sobre receber o perdão foram utilizados os descritores: "receber o perdão" e "buscar o perdão", em português e inglês. Após a aplicação dos critérios de inclusão e exclusão foram selecionados 12 artigos. Destacou-se a predominância do interesse na investigação da motivação para receber o perdão, sendo este um dos objetivos da maioria dos estudos revisados. Estes estudos contribuíram para o conhecimento da atitude para receber o perdão ao estabelecer os fatores, condições ou variáveis que favorecem a motivação para o pedido de desculpas ou para buscar receber o perdão. No que se refere ao autoperdão, foi utilizado o descritor "autoperdão", em português e inglês. Foram selecionados 54 artigos após a aplicação dos critérios de inclusão e exclusão. Parte considerável dos estudos buscou delimitar e diferenciar os processos de autoperdão genuíno e de pseudo-autoperdão. Estes estudos procuraram estabelecer como é possível se perdoar genuinamente e exploraram as variáveis que tem um papel significativo nesse processo. Considera-se que as revisões realizadas poderão auxiliar estudos e atuações que busquem incentivar as atitudes para receber o perdão e para perdoar a si mesmo, possibilitando conhecimentos para que as atuações sejam mais efetivas.

Palavras-chave: Receber o Perdão, Autoperdão, Revisão Sistemática.

\section{Forgiveness from the Offender's Perspective: A Review of Systematics}

\begin{abstract}
This study carried out systematic reviews on the attitudes to receiving forgiveness and self-forgiveness. Considering the lack of studies and the relevance of the themes, the aim was to understand how these attitudes have been investigated in recent publications. The articles were collected through the Periódicos Capes portal, and studies published between 2006 and 2016 were selected. In the receiving forgiveness' review, the following descriptors were used: "receiving forgiveness" and "seeking forgiveness", in Portuguese and English. After applying the inclusion and exclusion criteria, 12 articles were selected. It was highlighted the predominance of interest in the investigation of the motivation to receiving forgiveness, being this one of the objectives of most of the studies reviewed. These studies contributed to the knowledge of the attitude to receive forgiveness by establishing the factors, conditions or variables that favor the motivation to apologize or to seek forgiveness. Regarding self-forgiveness, the descriptor "self-forgiveness", in Portuguese and English, was used. We selected 54 articles after applying the inclusion and exclusion criteria. A considerable part of the studies sought to delimit and differentiate the processes of genuine self-forgiveness and pseudo self-forgiveness. These studies sought to establish how it is possible to genuinely self-forgive and explore the variables that play a significant role in this process. It is considered that the revisions made may help studies and actions that seek to encourage attitudes to receive forgiveness and self-forgiveness, allowing knowledge to make the actions more effective.
\end{abstract}

Keywords: Receiving Forgiveness, Self-forgiveness, Systematic Review. 


\title{
El Perdón bajo la Perspectiva del Ofensor: una Revisión de la Sistemática
}

\begin{abstract}
Resumen: En este estudio se realizaron revisiones sistemáticas sobre las actitudes para recibir el perdón y para perdonar a sí mismo. Considerando la escasez de estudios y la relevancia de los temas, el objetivo fue conocer la forma en que estas actitudes fueron investigadas en las publicaciones recientes. El levantamiento de los artículos fue realizado a través del portal Periódicos Capes, siendo seleccionados estudios publicados entre 2006 y 2016. En la revisión sobre recibir el perdón se utilizaron los descriptores: "recibir el perdón”, y "buscar el perdón", en portugués e inglés. Después de la aplicación de los criterios de inclusión y exclusión se seleccionaron 12 artículos. Se destacó el predominio del interés en la investigación de la motivación para recibir el perdón, siendo éste uno de los objetivos de la mayoría de los estudios revisados. Estos estudios contribuyeron al conocimiento de la actitud para recibir el perdón al establecer los factores, condiciones o variables que favorecen la motivación para la disculpa o para buscar recibir el perdón. Con respecto al auto-perdón, se utiliza el descriptor de "autoperdón” en portugués y en inglés. Se seleccionaron 54 artículos después de la aplicación de los criterios de inclusión y exclusión. Una parte considerable de los estudios trató de delimitar y diferenciar los procesos de auto-perdón genuino y pseudo-auto-perdón. Estos estudios buscaron establecer cómo es posible auto-perdonar genuinamente y explotaron las variables que tienen un papel significativo en ese proceso. Se considera que las revisiones realizadas podrán auxiliar estudios y actuaciones que busquen incentivar las actitudes para recibir el perdón y para perdonar a sí mismo, posibilitando conocimientos para que las actuaciones sean más efectivas. Parte considerable de los estudios buscó delimitar y diferenciar los procesos de auto-perdón genuino y de pseudo-auto-perdón.
\end{abstract}

Palabras clave: Recibir el Perdón, Autoperdón, Revisión de la Sistemática.

Este trabalho consiste em uma revisão da literatura sobre as atitudes para receber o perdão e perdoar a si mesmo. O perdão interpessoal é compreendido como uma atitude moral que contribui para o bem-estar físico e emocional das vítimas e ofensores, sendo, inclusive, importante para a continuidade das relações sociais e a manutenção dos vínculos afetivos entre membros de uma família, amigos, colegas em ambientes de trabalho e mesmo entre grupos sociais com história de violência e preconceitos (Enright, \& Fitzgibbons, 2015). O interesse pela temática do perdão na Psicologia tem crescido ao longo dos anos, e Robert Enright é um dos teóricos que se destaca nesse cenário com propostas teóricas e metodológicas. Enright e o Grupo de Estudos sobre o Desenvolvimento Humano (1996) propõem o estudo do perdão em uma tríade: (1) oferecer o perdão - que adota a perspectiva das vítimas, (2) receber o perdão e (3) autoperdão - que adotam a perspectiva dos ofensores. A atitude para oferecer o perdão tem sido privilegiada nas pesquisas em Psicologia, havendo maior concentração de estudos baseados na perspectiva da vítima. No entanto, sendo o perdão definido como uma ati- tude que envolve a qualidade da relação passada e presente entre vítimas e ofensores, é igualmente importante ampliar os conhecimentos sobre as atitudes para receber o perdão e para perdoar a si mesmo.

A atitude para receber o perdão pode ser definida como: "uma orientação para reduzir a culpa e o remorso gerados por uma injustiça cometida e o desejo de ser reconhecido como digno de perdão; sentir a dor da vítima e aceitar seus julgamentos, afetos e comportamentos negativos, enquanto espera por julgamentos, afetos e comportamentos positivos" (Alencar, 2017).

De acordo com Enright (1996), uma atitude genuína para receber o perdão envolve, por parte dos ofensores, reconhecer a injustiça cometida, sentir remorso pelo comportamento injusto e respeitar a vítima, compreendendo que a decisão sobre o perdão pertence a ela e que ela pode necessitar de tempo para considerar se perdoa ou não. É importante que o ofensor compreenda e tenha empatia pela vítima, que sofre por consequência da injustiça cometida por ele.

No tocante ao autoperdão, segundo Holmgren (1998), para que ele ocorra genuinamente é fundamental que o ofensor reconheça e sinta culpa pela ofensa 
cometida, aceite seu erro e supere os sentimentos negativos, tendo como base o respeito a si mesmo e pela vítima. É necessário que o ofensor vivencie sentimentos negativos decorrentes da ofensa e, em seguida, elabore estratégias para não cometer erros semelhantes, assumindo um compromisso moral consigo mesmo. Nessa direção, Enright (1996) definiram o autoperdão como: "uma orientação para abrandar a culpa e o remorso após o reconhecimento do erro, favorecendo a compaixão, generosidade e amor direcionados ao self" (p. 116, tradução nossa). Perdoar a si mesmo envolve refletir e reconhecer o mal causado ao outro, assumir a responsabilidade pela dor do outro e se arrepender da ação injusta para, a partir disto, buscar uma reconciliação com o selfe, possivelmente, com o outro que foi ofendido injustamente.

Considerando a escassez de estudos e a relevância de se compreender as atitudes para receber o perdão e perdoar a si mesmo, a proposta desse artigo é fazer uma revisão da literatura sobre os temas como um ponto de partida para que avanços possam ocorrer nessas áreas de pesquisa na Psicologia. A revisão tem o objetivo de conhecer a forma como os estudos recentes vêm sendo desenvolvidos na literatura científica nacional e internacional, e assim agregar conhecimentos à compreensão da atitude moral do perdão. É importante esclarecer que, como se trata de um estudo de exploração de temáticas pouco trabalhadas, não foram estabelecidos critérios rígidos na seleção dos artigos revisados. A pretensão é de sistematizar os estudos existentes e discutir suas características, e para isso, foi delimitada apenas a necessidade de tratar o perdão sob o olhar da Psicologia. Para facilitar a análise sobre cada atitude, as produções sobre receber o perdão e perdoar a si mesmo foram analisadas separadamente.

\section{Revisão da Literatura sobre Receber o Perdão}

\section{Método e procedimentos}

O levantamento dos artigos foi realizado através do portal "Periódicos Capes" em abril de 2016, utilizando como descritores os termos "receber o perdão", "buscar o perdão", "receiving forgiveness" e "seeking forgiveness". A revisão englobou estudos teóricos e empíricos, sendo considerados teóricos aqueles que fundamentaram e discutiram a teoria sem realizar pesquisas empíricas; ao passo que foram considera- dos estudos empíricos aqueles que se basearam em teorias para verificar relações entre variáveis ou testar hipóteses. Os critérios de inclusão para seleção dos artigos foram: ser um estudo revisado por pares e publicado entre os anos de 2006 e 2016. Foram listados 33 trabalhos, dos quais 23 eram artigos revisados por pares. Após a leitura dos resumos foram adotados os seguintes critérios de exclusão: (1) não apresentar a atitude para receber o perdão entre as temáticas principais; (2) repetição da publicação; e (3) impossibilidade de acesso ao texto completo. Ao fim deste processo restaram 12 artigos indexados nas bases de dados: Science Direct $(n=6)$, Academic OneFile $(n=3)$, EBSCOhost $(n=1)$, Scopus $(n=1)$ e PubMed/MEDLINE $(n=1)$. Estes 12 estudos foram descritos e analisados considerando as perspectivas teóricas, os objetivos, as metodologias e os resultados encontrados.

\section{Resultados}

\section{Perspectivas teóricas dos estudos sobre receber o perdão}

Esta sessão tinha o propósito de apresentar as definições de receber o perdão e as abordagens teóricas adotadas nos estudos revisados. No entanto, verificou-se que não houve uma descrição clara da definição de receber o perdão ou da perspectiva teórica adotada nos estudos revisados, que se fundamentaram majoritariamente em resultados de estudos empíricos anteriores. Destaca-se, entretanto, que Worthington foi o autor mais citado nos estudos, porém de forma pontual, sem fazer referência direta a sua teoria sobre o perdão (Bassett, Bassett, Lloyd, \& Johnson, 2006; Bassett et al., 2011; Chiaramello, Sastre, \& Mullet, 2008; Exline, Prince-Paul, Root, Peereboom, \&Worthington Jr., 2012; Howell, Dopko, Turowski \& Buro, 2011; Howell, Turowski, \& Buro, 2012; Paleari, Regalia, \& Fincham, 2011; Riek, 2010; Stouten, \& Tripp, 2009; Wallace, Exline, \& Baumeister, 2008). Worthington, assim como Enright, concebe o perdão como um processo, porém enfatiza sua constituição emocional e motivacional, ressaltando a importância do vínculo entre esses dois componentes.

\section{Objetivos dos estudos sobre receber o perdão}

No que se refere aos objetivos dos estudos revisados, percebeu-se uma predominância do interesse na investigação da motivação para receber o perdão, 
sendo este um dos objetivos principais de oito dos 12 estudos revisados. Cinco deles focaram na motivação para receber o perdão sem tratar de relações interpessoais específicas (Bassett et al., 2006; 2011; Chiaramello et al., 2008; Neto, Mullet, Chiaramello, \& Suwartono, 2013; Riek, 2010), enquanto três estudos trataram especificamente da motivação para pedir desculpas, considerando este comportamento como um componente importante para receber o perdão, sendo um possível precursor do mesmo (Howell et al., 2011; 2012; Leunissen, De Cremer, \& Folmer, 2012). Esses estudos buscaram estabelecer quais fatores, condições ou variáveis favorecem a motivação para o pedido de desculpas ou para buscar receber o perdão.

Além disso, alguns desses estudos analisaram de forma específica o papel das emoções morais, como culpa, vergonha, tristeza, raiva etc., na motivação para pedir desculpas e para receber o perdão (Bassett et al., 2006; 2011; Chiaramello et al., 2008; Howell et al., 2012; Riek, 2010). Essas emoções foram avaliadas como respostas situacionais experienciadas em uma transgressão específica e também como disposição de resposta do participante em situações de transgressão.

É importante destacar, no entanto, que os estudos não limitaram seu interesse ao fator Motivação. Paleari et al. (2011), por exemplo, basearam-se na teoria da equidade para investigar o efeito da iniquidade ou desequilíbrio entre oferecer o perdão e receber o perdão no casamento, analisando como esse desequilíbrio afetaria o bem-estar psicológico e emocional do casal. Ainda tendo como suporte a teoria da equidade, o estudo de Stouten e Tripp (2009) buscou examinar como membros de um grupo reagem quando a norma de equidade é violada e alguém contribui menos do que os outros. O objetivo dos autores foi analisar o efeito do pedido de perdão na reação das pessoas à violação, avaliando se a importância do pedido seria dependente do status do ofensor.

Também foi objetivo de um dos estudos revisados a análise das consequências de receber o perdão, testando se o mesmo desencorajaria ou incentivaria a repetição das transgressões (Wallace et al., 2008). E houve, ainda, um único caso em que a atitude para receber foi investigada em conjunto com as demais atitudes do perdão: oferecer o perdão e perdoar a si mesmo (Exline et al., 2012). Esse estudo teve como objetivo examinar a relevância do perdão pra familiares de portadores de transtorno mental, porém sem fazer distinção entre os seus diferentes componentes.

\section{Metodologias utilizadas nos estudos sobre receber o perdão}

Com relação às metodologias utilizadas nos estudos revisados, observou-se que todos foram estudos empíricos, sendo as medidas realizadas em um único período de tempo ou em períodos muitos próximos (por exemplo, Bassett et al., 2006). Dentre esses estudos, verificou-se que apenas três trabalhos utilizaram delineamento experimental (Leunissen et al., 2012; Stouten, \& Tripp, 2009; Wallace et al., 2008). Os demais realizaram exclusivamente pesquisas de levantamento sem manipulação de variáveis (Bassett et al., 2006; 2011; Chiaramello et al., 2008; Exline et al., 2012; Howell et al., 2011; 2012; Neto et al., 2013; Paleari et al., 2011; Riek, 2010).

Quanto aos participantes, percebe-se que na última década as pesquisas sobre receber o perdão trabalharam majoritariamente com estudantes universitários. Apenas duas das pesquisas descritas propuseram um estudo com uma população específica: familiares de pessoas portadoras de transtorno mental (Exline et al., 2012) e casais (Paleari et al., 2011).

\section{Resultados dos estudos sobre receber o perdão}

Conforme relatado anteriormente, apenas um estudo investigou o perdão sem distinguir entre as suas diferentes atitudes: oferecer o perdão, receber o perdão e perdoar a si mesmo (Exline et al., 2012). Esse estudo encontrou uma relação positiva entre ofensas não resolvidas e sintomas depressivos nos participantes, e mesmo sem tratar da atitude para receber o perdão de forma específica, esse resultado contribuiu para a reafirmação da relação entre perdão e depressão, tradicionalmente apontada pela literatura da área.

Nos demais estudos revisados, a atitude para receber o perdão foi tratada de forma clara, sendo distinta das outras atitudes do perdão ou de qualquer outra variável. Entre os estudos que tiveram como objetivo a motivação para receber o perdão, merecem destaque os trabalhos de Bassett et al. (2006) e Chiaramello et al. (2008), que avaliaram a estrutura fatorial dessa motivação e encontraram uma estrutura de três fatores na disposição para buscar receber o perdão. Os fatores encontrados, em cada um dos estudos, representam diferentes graus de motivação e podem ser considerados similares, de forma que: Dureza no coração (Bassett et al., 2006) e Inabilidade 
de procurar o perdão (Chiaramello et al., 2008) referem-se ao estado em que há menor motivação para buscar o perdão; Busca pela verdade (Bassett et al., 2006) e Sensibilidade às circunstâncias (Chiaramello et al., 2008) representam o estado em que o ofensor já considera a possibilidade, mas está avaliando as condições para decidir se deve ou não buscar o perdão; e, por fim, Busca pelo perdão (Bassett et al., 2006) e Procura incondicional do perdão (Chiaramello et al., 2008) referem-se ao estado em que há forte motivação para buscar o perdão, independentemente de qualquer condição. Essa estrutura trifatorial foi testada e corroborada por Neto et al. (2013), que confirmaram a confiabilidade do modelo em diferentes culturas (Portugal, Brasil, Indonésia, Angola e Moçambique).

Ainda na temática dos fatores relacionados à motivação para pedir desculpas e para receber o perdão, verificou-se que a culpa foi a emoção que recebeu maior destaque nos estudos que investigaram o papel das emoções morais, e foi possível observar que os autores também se interessaram por compará-la com outras emoções (Bassett et al., 2006; 2011; Howell et al., 2012). Apesar das distintas formas de análise, ressalta-se nesta revisão o resultado comum encontrado nos estudos acima citados: a vergonha como uma emoção que dificulta a procura do perdão, e a culpa como uma emoção que a facilita. Os estudos reiteraram a concepção de que a vergonha é uma emoção autocentrada, em que o sentido do Eu é alterado e a pessoa se sente sem valor, enquanto a culpa é uma emoção que volta a atenção para um comportamento específico, e não há uma desvalorização de si mesmo, pois apenas a ação é considerada repreensível. Assim, enquanto a vergonha levaria ao desejo de fuga, a culpa levaria ao desejo de reparação. Este resultado se manteve tanto em termos situacionais como em termos disposicionais.

Outras variáveis também foram apontadas pelos estudos como relacionadas à motivação para buscar receber o perdão e para se desculpar. As correlações positivas incluíram: amabilidade (Chiaramello et al., 2008; Howell et al., 2011), nível intelectual (Chiaramello et al., 2008), arrependimento e preocupação (Bassett et al., 2006), índices de funcionamento adaptativo, como compaixão, bem-estar, autoestima e aceitação (Howell et al., 2011), e variáveis situacionais, como responsabilidade sobre a ofensa, severidade da ofensa, proximidade da relação e ruminação (Riek, 2010). As relações com as variáveis situacionais foram mediadas pelo sentimento de culpa, reafirmando o papel positivo dessa emoção na promoção da atitude para receber o perdão. Em contrapartida, as correlações negativas englobaram: raiva (Chiaramello et al., 2008; Riek, 2010), ansiedade, cinismo, tendências paranoicas, tendências autopunitivas e neurose (Chiaramello et al., 2008). Ainda, foi analisado o papel da orientação temporal, verificando que pessoas voltadas para o futuro têm maior motivação para buscar receber o perdão do que aquelas que são presas ao passado (Chiaramello et al., 2008).

Ainda na temática da motivação, o estudo de Leunissen et al. (2012) propôs a investigação da chamada "motivação instrumental" para receber o perdão, que condiciona a motivação do ofensor à percepção da probabilidade de disposição da vítima para perdoar o mesmo. Os resultados mostraram uma motivação significativamente maior para pedir desculpas quando a chance da vítima desculpar era percebida como alta. Essa concepção da motivação para receber o perdão se diferencia dos demais estudos e na seção seguinte discutir-se-á o porquê de ela ser incompatível com a visão de uma atitude genuína.

Entre os estudos que se basearam na teoria da equidade, há o estudo de Paleari et al. (2011) que buscou verificar o efeito da iniquidade entre oferecer o perdão e receber o perdão no casamento. Os resultados mostraram que os homens perdoaram mais do que foram perdoados, o que representa uma iniquidade entre o casal. Porém, com relação ao impacto desta iniquidade para a satisfação com o casamento e bem-estar emocional, este estudo revelou que a percepção de iniquidade afetou negativamente o bem-estar emocional e relacional das esposas, relação esta que não se estabeleceu com os maridos.

No estudo de Stouten e Tripp (2009), por sua vez, foram utilizados dilemas sociais para analisar o efeito do pedido de perdão na reação de membros de um grupo à violação da norma de equidade, avaliando se haveriam diferenças quando o líder ou outro membro do grupo (considerado um igual) era o responsável pela violação de contribuir menos do que os demais membros. Com seus resultados, os autores constataram que a importância do pedido de desculpas foi dependente do status do ofensor. Entre os iguais, o pedido de desculpas foi necessário e eficiente para atenuar as emoções negativas e aumentar a possibilidade de perdão da vítima, ou seja, a probabilidade de receber o perdão. Entretanto, quando o violador 
era um líder, o papel do pedido de desculpas não foi igualmente importante. Os líderes foram perdoados mais facilmente e foram menos percebidos como violadores, sendo possível inferir que, devido a sua posição de poder, tenham sido considerados como detentores do direito de contribuir menos.

Por fim, para verificar as consequências de receber o perdão no incentivo a repetição das transgressões, Wallace et al. (2008) utilizaram medidas comportamentais e de autorrelato, buscando com isso uma análise mais completa dessa relação. Em seus resultados, receber o perdão foi preditor de uma maior motivação para o arrependimento, diminuindo a disposição para ofender novamente a mesma pessoa, de maneira que a maioria dos participantes se mostrou menos propensa a repetir as ofensas quando havia recebido o perdão da vítima.

\section{Discussão}

A revisão da literatura permitiu constatar que os estudos recentes sobre receber o perdão, além de escassos, não apresentam claramente o conceito dos autores sobre essa variável, tendo pouca relação com abordagens teóricas. Considera-se que essa característica pode ser associada ao fato de todos os estudos serem empíricos, podendo-se inferir uma maior preocupação dos autores com questões metodológicas e com a discussão de resultados em detrimento do esclarecimento teórico. No entanto, essa escolha é considerada problemática, pois a concepção teórica do perdão influencia substancialmente a forma de estudá-lo, sendo essa delimitação muito importante tanto para o autor, ao realizar seu estudo empírico, quanto para o leitor, na compreensão e análise crítica dos estudos.

No que se refere aos objetivos dos estudos revisados, destaca-se a prevalência do interesse pelo fator Motivação. Considera-se que tais investigações são de grande valia para estruturação epistemológica da atitude para receber o perdão e, sobretudo, para a promoção da mesma, pois elas possibilitam estabelecer os aspectos que devem ser foco de atenção no trabalho com ofensores. Ainda, é preciso considerar que, mesmo que de forma indireta, Worthington foi o autor mais referenciado nos estudos revisados. Sendo este um teórico que valoriza a constituição motivacional do perdão, é possível que haja relação com o fato de a motivação ter constituído o interesse predominante nos estudos sobre receber o perdão. No entanto, essa relação não pode ser confirmada devido à ausência de delimitação do referencial teórico dos estudos.

Ressalta-se, porém, que especificamente no estudo de Leunissen et al. (2012) a motivação o para receber o perdão foi abordada de forma diferente e contraditória. A denominada "motivação instrumental" parte de um forte interesse do ofensor em proteger a si mesmo e só procurar o perdão quando este se mostra "seguro", isto é, buscar o perdão quando nota que a vítima está disposta a oferecê-lo. Entretanto, quando o perdão é compreendido e vivenciado como uma atitude moral, ele não está condicionado a consequências externas. A genuína procura pelo perdão é uma atitude em que a pessoa escolhe reconhecer o erro e tenta repará-lo, independentemente da resposta do outro (Enright, \& Fitzgibbons, 2015).

Estudos como o de Paleari et al. (2011) e Stouten e Tripp (2009) também merecem ser destacados, pois chamam atenção para o aspecto relacional do perdão ao tratarem, respectivamente, dos efeitos da iniquidade nas relações conjugais e do status de poder do ofensor. Pode-se dizer que esses estudos reafirmaram a importância de sempre considerar todos os personagens envolvidos ao se trabalhar com o perdão, pois a forma como cada um reage trará implicações para as vivências individuais dos processos e é preciso considerar o possível impacto dos diferentes níveis de relacionamento.

Ainda, destaca-se o estudo de Wallace et al. (2008), que avaliou as consequências de receber o perdão. Essa temática é bastante discutida por defensores e críticos do perdão que discordam sobre seu papel em reiterar ou não o comportamento ofensivo (Enright \& Fitzgibbons, 2015). O resultado encontrado por Wallace et al. (2008) mostra que receber o perdão pode ter consequências positivas não só para o ofensor, mas também para a vítima e para a relação entre eles, desmistificando, assim, a ideia de que perdoar favorece a repetição da ofensa. Informações como esta estimulam o estudo sobre receber o perdão e corroboram a importância de trabalhos que busquem incentivá-lo.

Diante dos resultados desta revisão, verifica-se que a predominância do interesse das pesquisas pelo fator Motivação trouxe importantes contribuições para o conhecimento da atitude para receber o perdão. Porém, existem ainda muitos caminhos a serem explorados, especialmente por esta ser uma área recente de estudos. É importante expandir os 
conhecimentos sobre essa atitude, não só ampliando a quantidade de estudos, mas também a investigando com maior diversidade de delineamentos e populações, pois isso tornará mais abrangente e representativo o que se sabe sobre receber o perdão.

Por fim, é importante salientar a ausência de pesquisas brasileiras entre os estudos revisados. Verifica-se que a temática do perdão não é amplamente explorada no Brasil e o foco dos estudos têm sido as vítimas. Mostra-se, pois, relevante o desenvolvimento de pesquisas que considerem os ofensores, para avaliar como essas relações se estabelecem com a população brasileira.

\section{Revisão da Literatura sobre Autoperdão}

\section{Método e Procedimentos}

Os artigos desta revisão foram pesquisados através do portal Periódicos Capes em abril de 2016 e foram utilizados como descritores os termos "autoperdão" e "self forgiveness". Assim como na revisão anterior, foram contemplados estudos teóricos e empíricos. Foram elencados 113 artigos que atenderam aos critérios de inclusão de ser um estudo revisado por pares e publicado entre os anos de 2006 e 2016. Em seguida foi realizada a leitura dos resumos, sendo aplicados os seguintes critérios de exclusão: (1) não apresentar a atitude para o autoperdão entre as temáticas principais, (2) não ter relação com a Psicologia, (3) repetição da publicação e (4) impossibilidade de acesso ao texto completo. Ao fim deste processo restaram 54 artigos que foram revisados e analisados neste estudo. Estes estavam indexados nas bases de dados: Academic OneFile $(n=13)$, EBSCOhost $(n=12)$, SprigerLink ( $n$ $=8)$, Science Direct $(n=8)$, Sage Journals (4), Scopus $(n=1)$ e Ovid MEDLINE $(n=1)$. Sete artigos foram, ainda, obtidos através da Wiley Online Library que permitiu o acesso direto a revista na qual se encontra. As descrições e análises dos estudos foram realizadas considerando as perspectivas teóricas, os objetivos, as metodologias e os resultados encontrados.

\section{Resultados}

Perspectivas teóricas dos estudos sobre autoperdão

No que diz respeito às perspectivas teóricas utilizadas para o estudo do autoperdão, destaca-se que apenas 15 estudos estabeleceram claramente a defini- ção de autoperdão adotada, sendo grande a variedade de conceitos e teorias. A definição de Enright (1996) foi a mais prevalente (Day, Gerace, Wilson, \& Howells, 2008; Dixon, Earl, Lutz-Zois, Goodnight, \& Peatee, 2014; Exline, Root, Yadavalli, Martin, \& Fischer, 2011; Fisher, \& Exline, 2010; Friedman et al., 2007; Kim, \& Enright, 2014; Ursúa, \& Echegoyen, 2015; Wenzel, Woodyatt, \& Hedrick, 2012), seguida pela definição de Hall e Fincham (2005), que concebem o autoperdão como a diminuição da motivação para autopunição e retaliação contra si mesmo, bem como o aumento da motivação para benevolência em relação a si (Hall, \& Fincham, 2008; Krause, 2015; McConnell, \& Dixon, 2012; Rangganadhan, \& Todorov, 2010; Wohl, Pychyl, \& Bennett, 2010; Woodyatt, \&Wenzel, 2013a). Houve, ainda, a proposição de uma definição própria para o autoperdão no estudo de Cornish e Wade (2015), que criticaram as definições anteriores por considerar que são centradas apenas no ofensor e propuseram incluir na definição de autoperdão componentes voltados para vítima, a saber, a reparação da ofensa e a redução da probabilidade de cometer ofensa semelhante. Nos demais estudos, a visão conceitual dos autores foi explicitada a partir de informações obtidas em estudos anteriores, sem delimitar uma perspectiva teórica específica.

Apesar da diversidade de definições e da ausência de concordância sobre o que é o autoperdão, já existe na literatura um maior consenso na delimitação daquilo que ele não é. Houve uma clara distinção entre autoperdão e outros comportamentos de defesa, como negar, minimizar ou justificar a ofensa. Esses comportamentos podem ser denominados de pseudo-autoperdão, uma vez que não há o reconhecimento do erro e a aceitação da responsabilidade, que são fundamentais para o autoperdão genuíno que, por sua vez, requer tempo e esforço (Cornish, \&Wade, 2015; Exline et al., 2011; Fisher, \& Exline, 2006; 2010; Kim, \& Enright, 2014; Robb III, 2007; Ursúa \& Echegoyen, 2015; vanOyen-Witvliet et al., 2011; Wenzel et al., 2012; Wohl \& McLaughlin, 2014; Woodyatt \& Wenzel, 2013a, 2013b, 2014).

A aceitação da responsabilidade foi, inclusive, citada nos estudos revisados como um dos componentes fundamentais para que o autoperdão seja genuíno (Cornish \& Wade, 2015; Exline et al., 2011; Fisher \& Exline, 2006, 2010; Griffin, Lavelock \& Worthington Jr., 2014; Kim, \& Enright, 2014; McConnell, Dixon, \& Finch, 2012; Robb III, 2007; Ursúa, \& Echegoyen, 2015; Oyen-Witvliet et al., 2011; Wenzel et al., 
2012; Wohl, \& McLaughlin, 2014; Wohl et al., 2010; Woodyatt, \& Wenzel, 2013a;b; 2014; Worthington, \& Langberg, 2012). Afirmou-se nesses estudos que é preciso, inicialmente, reconhecer a injustiça cometida, assumir a responsabilidade e vivenciar as emoções negativas oriundas dela, para em seguida elaborar essa ofensa e reconquistar a autoaceitação, apesar do erro cometido.

Embora essa distinção seja clara na teoria, os estudos empíricos têm apontado a dificuldade em diferenciar o autoperdão genuíno e o pseudo-autoperdão (Cornish, \& Wade, 2015; Fisher, \& Exline, 2006; 2010; Ursúa; \& Echegoyen, 2015; Wenzel et al., 2012; Woodyatt \& Wenzel, 2013a;b). Os autores atribuíram essa limitação ao fato de os estudos medirem o autoperdão apenas pelo estado final do processo. Como o pseudo-autoperdão e o autoperdão genuíno levam ao mesmo resultado, que se caracteriza pela diminuição da autopunição e o aumento do sentimento positivo em relação a si mesmo, não é possível diferenciá-los focando apenas no resultado. Essa imprecisão justificaria os resultados de estudos, como o de Strelan (2007a), que associam o autoperdão a pessoas narcisistas e egocêntricas, que não sentem culpa ou arrependimento pelo ato injusto cometido.

\section{Objetivos dos estudos sobre autoperdão}

Considerando que é no processo que o autoperdão genuíno e o pseudo-autoperdão se diferenciam, dez estudos tiveram como objetivo a investigação e a delimitação desses processos (Cornish, \& Wade, 2015; Fisher, \& Exline, 2006; 2010; Hall, \& Fincham, 2008; Kim, \& Enright, 2014; McConnell et al., 2012; Ursúa, \& Echegoyen, 2015; Wenzel et al., 2012; Woodyatt, \& Wenzel, 2013a;b; 2014). Estes estudos buscaram estabelecer como é possível se perdoar genuinamente e exploraram as variáveis que tem um papel significativo ao longo desse processo. De forma mais específica, destaca-se o estudo de Cornish e Wade (2015), que teve como objetivo a proposição de um novo modelo de processo terapêutico para o autoperdão genuíno, e o estudo Woodyatt e Wenzel (2013a), que propôs um modelo que diferenciou as possíveis respostas do ofensor após a injustiça.

Alguns estudos investigaram, ainda, a relação específica entre o autoperdão e determinadas variáveis, como ideação e/ou comportamento suicida (Cheavens, Cukrowicz, Hansen, \& Mitchell, 2016; Hirsch, Webb, \& Jeglic, 2011; 2012; Nsamenang, Webb,
Cukrowicz, \& Hirsch, 2013), submissão à violência íntima (Davidson, Lozano, Cole, \& Gervais, 2015), procrastinação (Wohl et al., 2010), religião (Griffin et al., 2014; McConnell, \& Dixon, 2012) etc.

Entre os estudos revisados também houve interesse nos possíveis efeitos do autoperdão para a saúde física (Webb, Phillips, Bumgarner, \& Conway-Williams, 2013a; Wilson, Milosevic, Carroll, Hart, \& Hibbard, 2008) e mental (Fisher; \& Exline, 2006; Krause, 2015; Macaskill, 2012; Nsamenang et al., 2013; Romero et al., 2006; Webb et al., 2013a; Webb, Hirsch, Conway-Williams, \& Brewer, 2013b) do ofensor.

Os estudos revisados centraram-se, majoritariamente, nos aspectos positivos do autoperdão. Entretanto, três deles voltaram-se para um possível aspecto negativo do autoperdão, focando nas situações em que o mesmo poderia não ser apropriado (Squires, Sztainert, Gillen, Caouette, \& Wohl, 2012; Wohl, \& McLaughlin, 2014; Wohl, \& Thompson, 2011). Ainda, há o estudo de Vitz e Meade (2011) que realizaram uma análise crítica sobre os potenciais problemas do autoperdão, enquanto o estudo de Kim e Enright (2014) apresenta uma defesa teológica e psicológica do autoperdão, respondendo críticas contra o mesmo, incluindo as realizadas por Vitz e Meade (2011).

\section{Metodologias utilizadas nos estudos sobre autoperdão}

No que se refere às metodologias aplicadas nos estudos, percebe-se que, dos 54 artigos revisados, 11 foram de natureza teórica (Cornish, \&Wade, 2015; Day et al., 2008; Fisher, \& Exline, 2010; Griffin et al., 2014; Hong, \& Jacinto, 2012; Kim, \& Enright, 2014; Robb III, 2007; Ursúa, \& Echegoyen, 2015; Vitz, \& Meade, 2011; Wohl, \& McLaughlin, 2014; Worthington, \& Langberg, 2012), sendo dominante a pesquisa empírica nos estudos recentes sobre autoperdão. Nota-se que entre os estudos empíricos analisados, dois foram estudos de caso (Hong, \& Jacinto, 2012; Lander, 2012) e cinco foram longitudinais, porém a maioria destes compreendeu períodos que podem ser considerados restritos, sendo o ofensor acompanhado por 11 dias (Wenzel et al., 2012; Woodyatt \& Wenzel, 2013a;b) ou sete semanas (Hall, \& Fincham, 2008) após a ocorrência da ofensa. Apenas o estudo de Krause (2015) compreendeu período maior, realizando rodadas de entrevista entre os anos de 2007 e 2008.

Ainda, prevaleceram entre os estudos empíricos revisados as pesquisas de levantamento, enquanto 
apenas cinco estudos fizeram uso de delineamento experimental (Exline et al., 2011; Oyen-Witvliet et al., 2011; Wenzel et al., 2012; Woodyatt, \& Wenzel, 2013a; 2014). Em ambos os casos o autoperdão tem sido avaliado quase que exclusivamente através de medidas explícitas de autorrelato. Considerando que este tipo de medida apresenta fraquezas pelo longo tempo de resposta que permite a interveniência de vieses, os estudos de Bast e Barnes-Holmes (2014; 2015a;b), Bast, Barnes-Holmes e Barnes-Holmes (2015) e Bast, Linares, Gomes, Kovac, e Barnes-Holmes (2016) objetivaram desenvolver e empregar medidas implícitas de autoperdão, utilizando o Procedimento Implícito de Avaliação Relacional ${ }^{1}$. As medidas implícitas demandam uma resposta rápida do participante e são apresentadas pelos autores como uteis para complementar as medidas explícitas de autoperdão.

Quanto aos participantes, observa-se que, assim como na revisão anterior, os estudos recentes sobre o autoperdão também têm trabalhado predominantemente com estudantes universitários, até mesmo para tratar de questões específicas como problemas com álcool (Webb et al., 2013a), desordem alimentar (Feibelman, \& Turner, 2015), sintomas depressivos (Hirsch et al., 2011), personalidade boderline (Law, \& Chapman, 2015), problemas com jogo (Squires et al., 2012), vício em cigarros (Wohl, \& Thompson, 2011) e vivência de violência íntima (Davidson et al., 2015). Apesar de em menor quantidade, foram encontrados estudos que trabalharam com populações específicas e não universitárias, como idosos (Cheavens et al., 2016; Ingersoll-Dayton, Torges, \& Krause, 2010; Krause, 2015), mulheres com câncer de mama (Friedman et al., 2007; Romero et al., 2006), minorias sexuais LGBTQ (Greene, \& Britton, 2013), adolescentes que praticam ou praticaram autoinjúria (Westers, Rehfuss, Olson, \& Biron, 2012) e estudos de casos com pacientes com doença crônica (Hong, \& Jacinto, 2012) e desordem alimentar (anorexia) (Lander, 2012).

\section{Resultados dos estudos sobre autoperdão}

Entre os estudos que tiveram como objetivo a investigação e a delimitação dos processos de pseudo-autoperdão e autoperdão genuíno, serão descritos, inicialmente, os resultados encontrados pelo único estudo que investigou diretamente o processo psicológico da atitude de pseudo-autoperdão (Woodyatt, \&
Wenzel, 2013b). Os autores afirmaram que o pseudo-autoperdão pode não ser uma atitude negativa deliberada, mas sim uma resposta defensiva natural nos momentos em que o ofensor sente sua pertença ao grupo ameaçada devido à injustiça por ele cometida. Para testar a hipótese, realizaram um estudo laboratorial, em que manipularam a ameaça à pertença, e um estudo longitudinal, em que acompanharam os participantes 11 dias após ter sido cometida uma injustiça. Em seus resultados, o pseudo-autoperdão se relacionou positivamente com a percepção de respostas hostis da vítima, porém quando a resposta da vítima ocorreu de forma respeitosa, a relação com o pseudo-autoperdão foi negativa. Apesar de buscarem entender o processo do pseudo-autoperdão, esses autores não defenderam que esta seja a forma mais adequada de lidar com a injustiça cometida, e salientaram que o processamento efetivo de uma ofensa deve envolver a aceitação de responsabilidade sobre ela.

Com relação aos estudos que se voltaram a compreender o autoperdão genuíno, tem sido enfatizado o papel do autoperdão para a redução das emoções negativas associadas a ofensa como culpa, vergonha, remorso e/ou autocondenação. Porém, também foi avaliado o papel exercido por essas emoções negativas no autoperdão, sendo estabelecidas relações similares às referidas nos estudos sobre receber o perdão (Cornish, \&Wade, 2015; Davidson et al., 2015; Exline et al., 2011; Fisher, \& Exline, 2006; 2010; Greene, \& Britton, 2013; Hall, \& Fincham, 2008; Macaskill, 2012; Rangganadhan, \& Todorov, 2010). Esses estudos mostraram que culpa e remorso desempenham papéis mais úteis para o autoperdão, pois têm como foco a ação cometida e a preocupação com a vítima, e por isso motivam tentativas de restauração, ao passo que a vergonha e a autocondenação são emoções globais que afetam a imagem que a pessoa tem de si mesma e são propensas a gerar respostas de fuga e/ ou de autoproteção.

Uma visão diferenciada sobre a vergonha foi apresentada por Woodyatt e Wenzel (2014), que alertaram sobre os perigos de não vivenciar essa emoção após cometer uma ofensa. De acordo com esses autores, a vergonha se associa a comportamentos de defesa apenas quando a ameaça de rejeição é grande e a reparação de sua causa é difícil. Sendo assim, eles alegam que quando a reparação do ato é possível,

\footnotetext{
${ }^{1}$ Termo original na língua inglesa: Implicit Relational Assessment Procedure (IRAP).
} 
a vergonha pode atuar como sua motivação, o que resultaria em um papel relevante para o autoperdão.

A habilidade de manejar e de regular emoções vem sendo apontada na literatura como uma importante facilitadora do autoperdão (Hodgson, \& Wertheim, 2007; Lander, 2012). Fisher e Exline (2010) defenderam que é preciso ajudar o ofensor a caminhar da vergonha para culpa, considerando que esta última desempenha um melhor papel para o autoperdão. E no que se refere à culpa, Fisher e Exline (2010) e McConnell et al. (2012) ressaltaram a importância de se trabalhar a diminuição da mesma, especialmente por meio do incentivo a comportamentos conciliatórios, como pedir desculpas, confessar o erro ou buscar repará-lo.

O papel dos comportamentos conciliatórios também foi investigado e analisado por outros estudos (Cornish, \& Wade, 2015; Exline et al., 2011; Fisher, \& Exline, 2006; Hall, \& Fincham, 2008; McConnell et al., 2012; Oyen-Witvliet et al., 2011; Rangganadhan, \& Todorov, 2010; Ursúa, \& Echegoyen, 2015; Wenzel et al., 2012; Woodyatt, \& Wenzel, 2013a; 2014) que corroboraram sua influência positiva no autoperdão. Baseados no estudo de Hall e Fincham (2008), em que foi confirmada a relação entre comportamentos conciliatórios e autoperdão, McConnell et al. (2012) propuseram um modelo alternativo em que acrescentaram a mediação da percepção do perdão da vítima para a explicação dessa relação. A ideia defendida foi de que os comportamentos conciliatórios do ofensor poderiam favorecer o perdão da vítima, que uma vez percebido pelo ofensor, facilitaria também o autoperdão.

Ainda neste contexto, cabe destacar os estudos de Wenzel et al. (2012) e Woodyatt e Wenzel (2014) que explicaram a relação entre a aceitação da responsabilidade e o autoperdão por meio dos comportamentos conciliatórios e da sua relação com a reafirmação de valores. De acordo com os autores, ao cometer uma injustiça, o ofensor viola certos valores pessoais, e o reconhecimento do erro pode, inclusive, afetar negativamente a identidade moral do ofensor. Por isso, há uma relação negativa entre a aceitação da responsabilidade e uma autoestima positiva. Porém, quando o mesmo assume comportamentos conciliatórios, isso o leva a reafirmar seus valores anteriormente violados e sua identidade moral, o que contribui para a melhora de sua autoimagem e facilita o autoperdão.

Apesar de haver concordância com relação à percepção de um caminho ideal para o autoperdão
(Exline et al., 2011; Fisher, \& Exline, 2010; Ursúa, \& Echegoyen, 2015), foram Cornish e Wade (2015) que, baseados nas discussões da literatura sobre o tema, propuseram um novo modelo de processo terapêutico para o autoperdão. Esse processo, de acordo com os autores, é formado por quatro componentes sequenciais: (1) aceitação da Responsabilidade; (2) vivência do Remorso; (3) Restauração através de comportamentos conciliatórios e reafirmação de valores; e (4) Renovação da visão de si mesmo, reestabelecendo a aceitação, o respeito, a compaixão e a bondade. Esse modelo traz como principal inovação a inclusão do componente Restauração, que é voltado para o outro e tem um papel central no processo desses autores, tornando-o menos intrapsíquico.

Woodyatt e Wenzel (2013a) também avaliaram o autoperdão genuíno, propondo um modelo que diferenciou três possíveis respostas dos ofensores: autopunição, pseudo-autoperdão e autoperdão genuíno. Seus resultados demonstraram que o autoperdão genuíno foi a única resposta que, além de se associar a benefícios intrapessoais, como a melhora na autoestima e na autoconfiança, se associou também com benefícios interpessoais, ou seja, com respostas positivas também voltadas para a vítima, como maior empatia e desejo de reconciliação, e diminuição da evitação. Esses benefícios interpessoais do autoperdão também foram encontrados em outros estudos (Cornish, \& Wade, 2015; Day et al., 2008; Fisher, \& Exline, 2006; 2010; Rangganadhan, \& Todorov, 2010).

O papel das variáveis situacionais também foi avaliado, mesmo que de forma superficial, em alguns estudos. Essas variáveis foram relacionadas diretamente ao autoperdão ou a fatores importantes para o mesmo, como o remorso e os comportamentos conciliatórios: 1) a Severidade da Ofensa foi a variável mais prevalente nos estudos, sendo apontada sua associação negativa com o autoperdão (Hall, \& Fincham, 2008; McConnell et al., 2012) e sua associação positiva com o remorso e com os comportamentos conciliatórios (Fisher, \& Exline, 2006); 2) a Proximidade da Relação entre a vítima e o ofensor foi positivamente relacionada com o remorso (Fisher, \& Exline, 2006) e com os comportamentos conciliatórios (Exline et al., 2011; Fisher, \& Exline, 2006); e 3) o Tempo desde a Ocorrência da Ofensa se relacionou positivamente com o autoperdão (Hall, \& Fincham, 2008) e negativamente com os comportamentos conciliatórios (Exline 
et al., 2011). Apesar do pouco destaque dado a essas variáveis, mostra-se importante considerar o valor das mesmas para o autoperdão.

Os benefícios intrapessoais do autoperdão também foram apontados pelos artigos revisados. A melhora na autoimagem e na autoestima foram aspectos do autoperdão considerados por todos os estudos, mesmo que de forma indireta, uma vez que integram a própria definição do autoperdão, que é caracterizado pelo reestabelecimento do amor em relação a si mesmo, apesar do erro cometido. Além desse benefício, o autoperdão também se relacionou a melhorias para a saúde física (Webb et al., 2013a; Wilson et al., 2008), sendo, inclusive, indicados seus potenciais benefícios para o tratamento de câncer de mama (Friedman et al., 2007; Romero et al., 2006), alcoolismo (Webb et al., 2013b) e desordem alimentar (Feibelman, \& Turner, 2015; Lander, 2012).

As melhorias para a saúde mental do ofensor também foram verificadas nos estudos (Fisher, \& Exline, 2006; Krause, 2015; Macaskill, 2012; Nsamenang et al., 2013; Romero et al., 2006; Webb et al., 2013a;b), sendo apontada especificamente sua: associação negativa com depressão (Fisher, \& Exline, 2006; Hirsch et al., 2011; Ingersoll-Dayton et al., 2010; Law, \& Chapman, 2015; Nsamenang et al., 2013) e ansiedade (Fisher, \& Exline, 2006; Krause, 2015; Macaskill, 2012); e associação positiva com a qualidade de vida (Friedman et al., 2007; Romero et al., 2006) e com a satisfação com a vida (Fisher \& Exline, 2006; Krause, 2015; Macaskill, 2012).

Entre os estudos que investigaram a relação específica entre o autoperdão e determinadas variáveis, os resultados mostraram que o autoperdão associa-se negativamente com: a ideação e/ou comportamento suicida (Cheavens et al., 2016; Hirsch et al., 2011; 2012; Nsamenang et al., 2013); a autoinjúria não suicida (Westers et al., 2012); a submissão à violência íntima (Davidson et al., 2015); a autocondenação em militares (Worthington, \& Langberg, 2012); o perfeccionismo (Dixon et al., 2014; Kim, Johnson, \& Ripley, 2011); e a procrastinação (Wohl et al., 2010). Os resultados também apontaram as associações positivas do autoperdão com: a crença no mundo justo (Strelan, 2007b); a consciência plena ${ }^{2}$ (Webb et al., 2013a); a humildade (Krause, 2015); e a religião (Griffin et al., 2014; McConnell, \& Dixon, 2012).
Ainda, é importante destacar os estudos que se voltaram para o possível lado negativo do autoperdão, ressaltando as situações em que o mesmo poderia não ser apropriado (Squires et al., 2012; Wohl, \& McLaughlin, 2014; Wohl, \& Thompson, 2011). De acordo com esses autores, perdoar a si mesmo por um comportamento não saudável ou nocivo, como fumar ou apostar, pode ter um efeito prejudicial, como se associar a uma menor disposição para modificar tal comportamento. Wohl e McLaughlin (2014) ressaltaram a importância de se perdoar no momento certo, quando o comportamento nocivo já estiver encerrado, para que assim não prejudique o desejo de modificar o comportamento. Exline et al. (2011) e Fisher e Exline (2010) também enfatizaram a importância de não incentivar o autoperdão cedo demais, considerando que isso poderia atrapalhar a busca de reparação por parte do ofensor.

Buscando responder as críticas tecidas em estudos como o de Vitz e Meade (2011), Kim e Enright (2014) elaboraram uma defesa do autoperdão, em que os autores ressaltaram a importância de diferenciar pseudo-autoperdão do autoperdão genuíno, enfatizando o papel do reconhecimento do erro e da aceitação da responsabilidade. Destacaram, ainda, a importância de se distinguir as três formas de perdão (autoperdão, perdão de Deus, perdão da vítima), considerando que cada uma se desenvolve de maneira singular e independente, embora uma possa facilitar a outra. O estudo de Hall e Fincham (2008) confirmou a associação positiva entre a percepção de perdão da vítima e de Deus e o autoperdão.

Por fim, aponta-se a existência de estudos que enfatizaram que, enquanto a reconciliação é uma possibilidade para a atitude para oferecer o perdão, mas não uma condição, para o autoperdão a reconciliação é imprescindível (Hall, \& Fincham, 2008; Robb III, 2007; Ursúa, \& Echegoyen, 2015).

\section{Discussão}

A realização desta revisão evidenciou que o estudo do autoperdão tem crescido na Psicologia, o que denota fortalecimento desse campo de estudo e o reconhecimento da importância dessa atitude. $\mathrm{O}$ foco de parte dos estudos no autoperdão genuíno demonstra a maturidade desse campo, que já discute e procura solucionar problemas surgidos ao longo das aná-

${ }^{2}$ Termo original na língua inglesa: mindfulness. 
lises dessa atitude. As características que distinguem o autoperdão genuíno do pseudo-autoperdão, como o reconhecimento do erro e a aceitação da responsabilidade, legitimam o autoperdão como uma atitude moral, que apesar de auxiliar o ofensor em uma reconciliação consigo mesmo, não atua justificando seus atos e eximindo-o de sua responsabilidade.

A assunção de responsabilidade gera emoções negativas que, por sua vez, possuem um papel complexo. Por um lado, essas emoções são essenciais para que o autoperdão seja genuíno, mas elas também são um obstáculo para o mesmo, pelo menos inicialmente, pois quanto mais grave a ofensa e maiores as emoções negativas, maior será o esforço necessário para que o autoperdão seja alcançado. Por esse motivo foi relevante que parte dos estudos revisados tenha analisado quais variáveis têm importância na transição desse processo e na explicação dessa relação, ou seja, tenha buscado explicar como é possível perdoar a si mesmo de forma genuína.

Outro elemento que chamou atenção nos estudos revisados foi a ênfase no papel positivo dos comportamentos conciliatórios para o autoperdão. Faz-se importante ressaltar, no entanto, que esses comportamentos conciliatórios não devem ser encarados como meras compensações. Para que sejam moralmente elevados é preciso que esses comportamentos se originem do reconhecimento da responsabilidade e que se pautem primordialmente na preocupação com o outro, e não no desejo de se sentir melhor consigo mesmo. Defende-se que somente assim esses comportamentos poderão ser genuínos, sendo viável a sua relação com a reafirmação de valores, conforme defendido nos estudos de Wenzel et al. (2012) e Woodyatt e Wenzel (2014).

As análises relativas aos benefícios do autoperdão mostraram que, além das contribuições para melhorias intrapessoais, o autoperdão também se associa a benefícios interpessoais. Esses resultados fortalecem a visão de que o autoperdão genuíno não é uma atitude egoísta e de favorecimento do ofensor, pois pode gerar consequências positivas também para as vítimas. Essa característica enfraquece parte das críticas endereçadas ao autoperdão e pode ser considerada como um estímulo à promoção do mesmo.

A revisão realizada também revelou a preocupação de alguns estudiosos com o impacto negativo que o autoperdão poderia ter, caso seja alcançado cedo demais, pois isso poderia atrapalhar a busca pelo per- dão da vítima e a tentativa de reparação. No entanto, é importante ter em mente que, ao se tratar do autoperdão genuíno, a ideia de que o mesmo desencorajaria a mudança de comportamento e a preocupação com o outro não se sustenta, pois a pessoa se perdoa tendo consciência do erro que cometeu e de sua responsabilidade sobre ele, e não esquecendo, negando ou justificando o ato cometido. Além disso, a concepção de parte dos estudos revisados de que o autoperdão exige a reconciliação consigo mesmo é um importante aspecto desta atitude, que fortalece a importância dos comportamentos conciliatórios e da mudança de comportamento do ofensor como formas de recuperar a confiança em si mesmo para que seja possível essa reconciliação.

No que se refere aos delineamentos utilizados, percebe-se que os estudos longitudinais foram escassos e realizados em períodos muito curtos de tempo. Essa caraterística deve ser discutida e considerada em estudos futuros, pois como demonstraram Hall e Fincham (2008), as covariações com o autoperdão podem diferir ao longo do tempo e o foco momentâneo pode levar ao erro na análise do seu processo.

Além disso, verifica-se que, comparados com receber o perdão, os estudos com amostras populacionais específicas estão mais desenvolvidos no autoperdão. No entanto, chama atenção a predominância da participação de universitários, mesmo em estudos sobre assuntos específicos, como o alcoolismo e a desordem alimentar, por exemplo. Considera-se que o estudo do autoperdão também merece maior diversidade de participantes e delineamentos, pois somente dessa maneira os conhecimentos sobre esta atitude poderão se tornar cada vez mais completos. Ainda, assim como na revisão anterior, a ausência de estudos brasileiros sobre autoperdão fortalece a visão de que as dimensões do perdão referentes aos ofensores ainda precisam ser exploradas no nosso país.

\section{Considerações Finais}

A análise, avaliação e integração da literatura publicada permitiu aprofundar o entendimento sobre as atitudes para receber o perdão e para perdoar a si mesmo. A ausência de estudos brasileiros na revisão realizada mostra como essas formas de perdão ainda são pouco exploradas no território nacional. As informações da literatura internacional atestam a importância de as dimensões receber o perdão e autoper- 
dão, bem com os benefícios associados a elas. Desta maneira, este é um campo de estudo que se mostra promissor, sendo essencial avaliar até que ponto as teorias e modelos desenvolvidos se adequam à população nacional.

Além disso, também é importante partir para a aplicação do que já se sabe. Explorar teoricamente as dimensões receber o perdão e autoperdão tem grande valia, mas os estudos indicam que muitos ganhos podem ser advindos da promoção dessas atitudes, não somente para os ofensores, mas também para as vítimas e para as relações interpessoais de uma maneira geral. A presente revisão pode auxiliar estudos e atuações que busquem incentivar a atitude para receber o perdão e para perdoar a si mesmo, possibilitando conhecimentos para que a atuação prática seja mais efetiva.

Sabe-se, no entanto, que existem limitações no alcance desta revisão de literatura. Todos os estudos revisados foram obtidos através do Portal "Periódicos Capes", sendo provável a existência de produções científicas em bases de dados que não foram analisadas no presente estudo. Porém, considera-se que mesmo com limitações, este estudo realizou uma revisão da literatura relevante, que poderá ser uma ferramenta útil aos estudiosos do perdão.

\section{Referências}

Alencar, T. L. F. (2017). Intervenção para o perdão em apenados (Dissertação de mestrado). Universidade Federal da Paraíba, João Pessoa, PB, Brasil.

Bassett, R. L., Bassett, K. M., Lloyd, M. W., \& Johnson, J. L. (2006). Seeking forgiveness: Considering the role of moral emotions. Journal of Psychology and Theology, 34(2), 111-124. https://doi.org/10.1177/009164710603400201

Bassett, R. L., Pearson, E., Ochs, S., Brennon, J., Krebs, G., Burt, L. et al. (2011). Feeling bad: The different colors of remorse. Journal of Psychology and Christianity, 30(1), 51-70.

Bast, D. F., \& Barnes-Holmes, D. (2014). A first test of the implicit relational assessment procedure as a measure of forgiveness of self and others. The Psychological Record, 64(2), 253-260. https://doi.org/10.1007/s40732-014$0022-2$

Bast, D. F., \& Barnes-Holmes, D. (2015a). Developing the implicit relational assessment procedure (IRAP) as a measure of self-forgiveness related to failing and succeeding behaviors. The Psychological Record, 65(1), 189-201. https://doi.org/10.1007/s40732-014-0100-5

Bast, D. F., \& Barnes-Holmes, D. (2015b). Priming thoughts of failing versus succeeding and performance on the implicit relational assessment procedure (IRAP) as a measure of self-forgiveness. The Psychological Record, 65(4), 667-678. https:// doi.org/10.1007/s40732-015-0137-0

Bast, D. F., Barnes-Holmes, Y., \& Barnes-Holmes, D. (2015). Developing an individualized implicit relational assessment procedure (IRAP) as a potential measure of self-forgiveness related to negative and positive behavior. The Psychological Record, 65(4), 717-730. https://doi.org/10.1007/s40732-015-0141-4

Bast, D. F., Linares, I. M. P., Gomes, C., Kovac, R., \& Barnes-Holmes, D. (2016). The implicit relational assessment procedure (IRAP) as a measure of self-forgiveness: The impact of a training history in clinical behavior analysis. The Psychological Record, 66(1), 177-190. https://doi.org/10.1007/s40732-016-0162-7

Cheavens, J. S., Cukrowicz, K. C., Hansen, R., \& Mitchell, S. M. (2016). Incorporating resilience factors into the interpersonal theory of suicide: The role of hope and self-forgiveness in an older adult sample. Journal of Clinical Psychology, 72(1), 58-69. https://doi.org/10.1002/jclp.22230

Chiaramello, S., Sastre, M. T. M., \& Mullet, E. (2008). Seeking forgiveness: Factor structure, and relationships with personality and forgivingness. Personality and Individual Differences, 45(5), 383-388. https://doi.org/10.1016/j. paid.2008.05.009

Cornish, M. A., \& Wade, N. G. (2015). A therapeutic model of self forgiveness with intervention strategies for counselors. Journal of Counseling \& Development, 93(1), 96-104. https://doi.org/10.1002/j.1556-6676.2015.00185.x

Day, A., Gerace, A., Wilson, C., \& Howells, K. (2008). Promoting forgiveness in violent offenders: A more positive approach to offender rehabilitation? Aggression and Violent Behavior, 13(3), 195-200. https://doi.org/10.1016/j. avb.2008.03.004

Davidson, M. M., Lozano, N. M., Cole, B. P., \& Gervais, S. J. (2015). Relations between intimate partner violence and forgiveness among college women. Journal of Interpersonal Violence, 30(18), 3217-3243. https://doi. org/10.1177/0886260514555008 
Dixon, L. J., Earl, K. A., Lutz-Zois, C. J., Goodnight, J. A., \& Peatee, J. J. (2014). Explaining the link between perfectionism and self-forgiveness: The mediating roles of unconditional self-acceptance and rumination. Individual Differences Research, 12(3), 101-111.

Enright, R. D. (1996). Counseling within the forgiveness triad: On forgiving, receiving forgiveness, and self-forgiveness. Counseling and Values, 40(2), 107-126. https:// doi.org/10.1002/j.2161-007X.1996.tb00844.X

Enright, R. D., \& Fitzgibbons, R. P. (2015). [Helping clients forgive] forgiveness therapy: An empirical guide for resolving anger and restoring hope. Washington, DC: American Psychological Association.

Exline, J. J., Prince-Paul, M., Root, B. L., Peereboom, K. S., \& Worthington Jr., E. L. (2012). Forgiveness, depressive symptoms, and communication at the end of life: A study with family members of hospice patients. Journal of Palliative Medicine, 15(10), 1113-1119. https://doi.org/10.1089/jpm.2012.0138

Exline, J. J., Root, B. L., Yadavalli, S., Martin, A. M., \& Fisher, M. L. (2011). Reparative behaviors and self-forgiveness: Effects ofalaboratory-basedexercise. SelfandIdentity, 10(1),101-126.https://doi.org/10.1080/15298861003669565

Feibelman, J. L., \& Turner, L. A. (2015). Relationships between eating disorder symptomology and forgiveness among college students. Current Psychology, 34(1), 121-129. https://doi.org/10.1007/s12144-014-9245-2

Fisher, M. L., \& Exline, J. J. (2006). Self-forgiveness versus excusing: The roles of remorse, effort, and acceptance of responsibility. Self and Identity, 5(02), 127-146. https:// doi.org/10.1080/15298860600586123

Fisher, M.L., \&Exline, J. J. (2010). Moving toward selfforgiveness: Removing barriers related to shame, guilt, and regret. Social and Personality Psychology Compass, 4(8), 548-558. https://doi.org/10.1111/j.1751-9004.2010.00276.X

Friedman, L. C., Romero, C., Elledge, R., Chang, J., Kalidas, M., Dulay, M. F. et al. (2007). Attribution of blame, self-forgiving attitude and psychological adjustment in women with breast cancer. Journal of Behavioral Medicine, 30(4), 351-357. https://doi.org/10.1007/s10865-007-9108-5

Greene, D. C., \& Britton, P. J. (2013). The influence of forgiveness on lesbian, gay, bisexual, transgender, and questioning individuals' shame and self esteem. Journal of Counseling \& Development, 91(2), 195-205. https://doi. $\operatorname{org} / 10.1002 /$ j.1556-6676.2013.00086.x

Griffin, B. J., Lavelock, C. R., \&Worthington Jr., E. L. (2014). On earth as it is in heaven: Healing through forgiveness. Journal of Psychology \& Theology, 42(3), 252-259. https://doi.org/10.1177/009164711404200302

Hall, J. H., \& Fincham, F. D. (2005). Self-forgiveness: The stepchild of forgiveness research. Journal of Social and Clinical Psychology, 24(5), 621-637. https://doi.org/10.1521/jscp.2005.24.5.621

Hall, J. H., \& Fincham, F. D. (2008).The temporal course of self-forgiveness. Journal of Social and Clinical Psychology, 27(2), 174-202. https://doi.org/10.1521/jscp.2008.27.2.174

Hirsch, J. K., Webb, J. R., \&Jeglic, E. L. (2011). Forgiveness, depression, and suicidal behavior among a diverse sample of college students. Journal of Clinical Psychology, 67(9), 896-906. https://doi.org/10.1002/jclp.20812

Hirsch, J. K., Webb, J. R., \& Jeglic, E. L. (2012). Forgiveness as a moderator of the association between anger expression and suicidal behaviour. Mental Health, Religion \& Culture, 15(3), 279-300. https://doi.org/10.1080/1367467 6.2011 .571666

Hodgson, L. K., \& Wertheim, E. H. (2007). Does good emotion management aid forgiving? Multiple dimensions of empathy, emotion management and forgiveness of self and others. Journal of Social and Personal Relationships, 24(6), 931-949. https://doi.org/10.1177/0265407507084191

Holmgren, M. R. (1998). Self-forgiveness and responsible moral agency. The Journal of Value Inquiry, 32(1), 75-91. https://doi.org/10.1023/A:1004260824156

Hong, Y. J., \& Jacinto, G. A. (2012). Six step therapeutic process to facilitate forgiveness of self and others. Clinical Social Work Journal, 40(3), 366-375. https://doi.org/10.1007/s10615-011-0377-7

Howell, A. J., Dopko, R. L., Turowski, J. B., \& Buro, K. (2011). The disposition to apologize. Personality and Individual Differences, 51(4), 509-514. https://doi.org/10.1016/j.paid.2011.05.009

Howell, A. J., Turowski, J. B., \& Buro, K. (2012). Guilt, empathy, and apology. Personality and Individual Differences, 53(7), 917-922. https://doi.org/10.1016/j.paid.2012.06.021

Ingersoll-Dayton, B., Torges, C., \& Krause, N. (2010). Unforgiveness, rumination, and depressive symptoms among older adults. Aging \& Mental Health, 14(4), 439-449. https://doi.org/10.1080/13607860903483136 
Kim, J. J., \& Enright, R. D. (2014). A theological and psychological defense of self-forgiveness: Implications for counseling. Journal of Psychology \& Theology, 42(3), 260-268.

Kim, L. M., Johnson, J. L., \& Ripley, J. (2011). A “perfect” storm: Perfectionism, forgiveness, and marital satisfaction. Individual Differences Research, 9(4), 199-209.

Krause, N. (2015). Assessing the relationships among race, religion, humility, and self-forgiveness: A longitudinal investigation. Advances in Life Course Research, 24, 66-74. https://doi.org/10.1016/j.alcr.2015.02.003

Lander, I. (2012). Towards the incorporation of forgiveness therapy in healing the complex trauma of parental incarceration. Child and Adolescent Social Work Journal, 29(1), 1-19. https://doi.org/10.1007/s10560-011-0248-7

Law, K. C., \& Chapman, A. L. (2015). Borderline personality features as a potential moderator of the effect of anger and depressive rumination on shame, self-blame, and self-forgiveness. Journal of Behavior Therapy and Experimental Psychiatry, 46, 27-34. https://doi.org/10.1016/j.jbtep.2014.07.008

Leunissen, J. M., De Cremer, D., \& Folmer, C. P. R. (2012). An instrumental perspective on apologizing in bargaining: The importance of forgiveness to apologize. Journal of Economic Psychology, 33(1), 215-222. https://doi. org/10.1016/j.joep.2011.10.004

Macaskill, A. (2012). Differentiating dispositional self-forgiveness from other-forgiveness: Associations with mental health and life satisfaction. Journal of Social and Clinical Psychology, 31(1), 28-50. https://doi.org/10.1521/jscp.2012.31.1.28

McConnell, J. M., \& Dixon, D. N. (2012). Perceived forgiveness from god and self-forgiveness. Journal of Psychology and Christianity, 31(1), 31-39.

McConnell, J. M., Dixon, D. N., \& Finch, W. H. (2012). An alternative model of self-forgiveness. Journal of Social and Psychological Sciences, 5(2), 22-53.

Neto, F., Mullet, E., Chiaramello, S., \& Suwartono, C. (2013). Seeking forgiveness: Factor structure in samples from Latin America, Africa, Asia, and Southern Europe. Universitas Psychologica, 12(3), 663-669. https://doi. org/10.11144/Javeriana.UPSY12-3.sffs

Nsamenang, S. A., Webb, J. R., Cukrowicz, K. C., \& Hirsch, J. K. (2013). Depressive symptoms and interpersonal needs as mediators of forgiveness and suicidal behavior among rural primary care patients. Journal of Affective Disorders, 149(1-3), 282-290. https://doi.org/10.1016/j.jad.2013.01.042

Oyen-Witvliet, C., Hinman, N. G., Exline, J. J., \& Brandt, T. (2011). Responding to our own transgressions: An experimental writing study of repentance, offense rumination, self-justification, and distraction. Journal of Psychology and Christianity, 30(3), 223-238.

Paleari, F. G., Regalia, C., \& Fincham, F. D. (2011). Inequity in forgiveness: Implications for personal and relational well-being. Journal of Social and Clinical Psychology, 30(3), 297-324. https://doi.org/10.1521/jscp.2011.30.3.297

Rangganadhan, A. R., \& Todorov, N. (2010). Personality and self-forgiveness: The roles of shame, guilt, empathy and conciliatory behavior. Journal of Social and Clinical Psychology, 29(1), 1-22. https://doi.org/10.1521/jscp.2010.29.1.1

Riek, B. M. (2010). Transgressions, guilt, and forgiveness: A model of seeking forgiveness. Journal of Psychology and Theology, 38(4), 246-254. https://doi.org/10.1177/009164711003800402

Robb III, H. B. (2007). Treating anger with forgiveness may sometimes require reconciliation. Journal of Rational-Emotive \& Cognitive-Behavior Therapy, 25(1), 65-75. https://doi.org/10.1007/s10942-006-0030-5

Romero, C., Friedman, L. C., Kalidas, M., Elledge, R., Chang, J., \& Liscum, K. R. (2006). Self-forgiveness, spirituality, and psychological adjustment in women with breast cancer. Journal of Behavioral Medicine, 29(1), 29-36. https://doi.org/10.1007/s10865-005-9038-z

Squires, E. C., Sztainert, T., Gillen, N. R., Caouette, J., \& Wohl, M. J. (2012). The problem with self-forgiveness: Forgiving the self deters readiness to change among gamblers. Journal of Gambling Studies, 28(3), 337-350. https:// doi.org/10.1007/s10899-011-9272-y

Stouten, J., \& Tripp, T. M. (2009). Claiming more than equality: Should leaders ask for forgiveness? The Leadership Quarterly, 20(3), 287-298. https:// doi.org/10.1016/j.leaqua.2009.03.002

Strelan, P. (2007b). The prosocial, adaptive qualities of just world beliefs: Implications for the relationship between justice and forgiveness. Personality and Individual Differences, 43(4), 881-890. https://doi.org/10.1016/j. paid.2007.02.015 
Strelan, P. (2007a). Who forgives others, themselves, and situations? The roles of narcissism, guilt, self-esteem, and agreeableness. Personality and Individual Differences, 42(2), 259-269. https://doi.org/10.1016/j.paid.2006.06.017

Ursúa, M. P., \& Echegoyen, I. (2015). ¿̇Perdón a uno mismo, autoaceptación o restauraciónintrapersonal? Cuestiones abiertas em psicología del perdón. Papeles del psicólogo, 36(3), 230-237.

Vitz, P. C., \& Meade, J. M. (2011). Self-forgiveness in psychology and psychotherapy: A critique. Journal of Religion and Health, 50(2), 248-263. https://doi.org/10.1007/s10943-010-9343-x

Wallace, H. M., Exline, J. J., \& Baumeister, R. F. (2008). Interpersonal consequences of forgiveness: Does forgiveness deter or encourage repeat offenses? Journal of Experimental Social Psychology, 44(2), 453-460. https://doi. org/10.1016/j.jesp.2007.02.012

Webb, J. R., Hirsch, J. K., Conway-Williams, E., \& Brewer, K. G. (2013b). Forgiveness and alcohol problems: Indirect associations involving mental health and social support. Addiction Research \& Theory, 21(2), 141-153. https:// doi.org/10.3109/16066359.2012.703267

Webb, J. R., Phillips, T. D., Bumgarner, D., \& Conway-Williams, E. (2013a). Forgiveness, mindfulness, and health. Mindfulness, 4(3), 235-245. https:// doi.org/10.1007/s12671-012-0119-0

Wenzel, M., Woodyatt, L., \& Hedrick, K. (2012). No genuine self forgiveness without accepting responsibility: Value reaffirmation as a key to maintaining positive self-regard. European Journal of Social Psychology, 42(5), 617-627. https://doi.org/10.1002/ejsp.1873

Westers, N. J., Rehfuss, M., Olson, L., \& Biron, D. (2012). The role of forgiveness in adolescents who engage in nonsuicidal self-injury. The Journal of nervous and mental disease, 200(6), 535-541. https://doi.org/10.1097/ NMD.0b013e318257c837

Wilson, T., Milosevic, A., Carroll, M., Hart, K., \& Hibbard, S. (2008). Physical health status in relation to self-forgiveness and other-forgiveness in healthy college students. Journal of Health Psychology, 13(6), 798-803. https://doi. $\operatorname{org} / 10.1177 / 1359105308093863$

Wohl, M. J.,\& McLaughlin, K. J. (2014). Self forgiveness: The good, the bad, and the ugly. Social and Personality Psychology Compass, 8(8), 422-435. https://doi.org/10.1111/spc3.12119

Wohl, M. J., Pychyl, T. A., \& Bennett, S. H. (2010). I forgive myself, now I can study: How self-forgiveness for procrastinating can reduce future procrastination. Personality and Individual Differences, 48(7), 803-808. https://doi. org/10.1016/j.paid.2010.01.029

Wohl, M. J., \& Thompson, A. (2011). A dark side to self forgiveness: Forgiving the self and its association with chronic unhealthy behaviour. British Journal of Social Psychology, 50(2), 354-364. https://doi.org/10.1111/j. 2044-8309.2010.02010.x

Woodyatt, L., \& Wenzel, M. (2013a). Self-forgiveness and restoration of an offender following an interpersonal transgression. Journal of Social and Clinical Psychology, 32(2), 225. https://doi.org/10.1521/jscp.2013.32.2.225

Woodyatt, L., \& Wenzel, M. (2013b). The psychological immune response in the face of transgressions: Pseudo self-forgiveness and threat to belonging. Journal of Experimental Social Psychology, 49(6), 951-958. https://doi. org/10.1016/j.jesp.2013.05.016

Woodyatt, L., \&Wenzel, M. (2014). A needs-based perspective on self-forgiveness: Addressing threat to moral identity as a means of encouraging interpersonal and intrapersonal restoration. Journal of Experimental Social Psychology, 50, 125-135. https://doi.org/10.1016/j.jesp.2013.09.012

Worthington, E. L., \& Langberg, D. (2012). Religious considerations and self-forgiveness in treating complex trauma and moral injury in present and former soldiers. Journal of Psychology \& Theology, 40(4), 274-288. https://doi. org/10.1177/009164711204000403

\section{Thalita Fernandes Alencar}

Doutoranda em Psicologia pelo Programa de Pós-Graduação em Psicologia Social da Universidade Federal da Paraíba (UFPB), João Pessoa - PB. Brasil. Mestre em Psicologia Social pela UFPB.

E-mail: thalitaalays@hotmail.com

(iD https://orcid.org/0000-0003-3898-6242 


\section{Eloá Losano Abreu}

Professora Adjunto do Departamento de Psicologia da Universidade Federal da Paraíba (UFPB).

Email: eloalosano@gmail.com

(iD https://orcid.org/0000-0001-7712-6275

Endereço para envio de correspondência:

Rua Antônio Vieira da Silva, n 400, ap 1404 C. Bairro Jardim São Paulo. Cep: 58053-175. João Pessoa 凶 PB. Brasil.

Recebido 22/09/2017

Aceito 16/07/2018

Received 09/22/2017

Approved $07 / 16 / 2018$

Recibido 22/09/2017

Aceptado 16/07/2018

Como citar: Alencar, T. F., \& Abreu, E. L. (2019). O perdão sob a perspectiva do ofensor: Uma revisão da sistemática. Psicologia: Ciência e Profissão, 39, 1-17. https://doi.org/10.1590/1982-3703003185662

How to cite: Alencar, T. F., \& Abreu, E. L. (2019). Forgiveness from the offender's perspective: A review of systematics. Psicologia: Ciência e Profissão, 39, 1-17. https://doi.org/10.1590/1982-3703003185662

Cómo citar: Alencar, T. F., \& Abreu, E. L. (2019). El perdón bajo la perspectiva del ofensor: Una revisión de la sistemática. Psicologia: Ciência e Profissão, 39, 1-17. https://doi.org/10.1590/1982-3703003185662 